\title{
Outcome after Fluoroscopic Guided Closed Reduction and Percutaneous Kirschner Wire Fixation of Lisfranc Joint Injuries
}

\author{
Shamim Ahmad Bhat*, Khurshid Ahmad Kangoo, Adnan Zahoor, Rameez Raja and Sami Jan
}

Government Medical College Srinagar, Srinagar, Jammu and Kashmir, India

\begin{abstract}
Twenty patients of Lisfranc joint injuries managed by flouroscopic guided closed reduction and percutaneous K-Wire fixation were evaluated for functional results with average follow up of 24 months. Males were $80 \%$ of total cases and road traffic accident represented cause of injury in $55 \%$ cases rest being due to fall. Majority of injuries were type B as per Myersons classification.

The average AOFAS-M score was 78 with $80 \%$ patients returning to their original occupation.

Early flouroscopic guided closed redusction and K-Wire stabilization of Lisfranc injuries is effective easy and reliable method of Lisfranc joint injury treatment with no secondary surgical procedure needed for implant removal and no cosmetic problem of scar formation
\end{abstract}

Keywords: Lisfranct fracture dislocation; Tarsometatarsal joint injuries; Closed reduction; Percutaneous pinning

\section{Introduction}

The tarsometatarsal joint injuries are also named as Lisfranc joint injuries after Jacques Lisfranc, a surgeon in Napoleon's army described an amputation through the joint for gangrenous injuries of the forefoot [1]. Fracture dislocations of the Lisfranc (tarsometatarsal) joints of foot are uncommon but serious injuries, with high potential for chronic disability. These injuries can easily be missed in the emergency department as radiographs may show only subtle incongruity of the joint [2] (Figures 1 and 2).

The satisfactory outcome of fracture dislocation of tarsometatarsal joints depend on early accurate diagnosis with prompt anatomic reduction, and stable internal fixation [3]. Thorough understanding of mechanism of injury combined with advances in technology, various options for surgical management range from percutaneous Kirschner wire (K-wire) fixations to primary arthrodesis. Several implants currently being utilized for fixation of these injuries include K-wires, screws, plates and suture anchors. Although the current trend for management of these fractures is towards open reduction and screw/Kirschner wire fixation [4,5]. Closed reduction and percutaneous Kirschner wire fixation has been reported in literature with acceptable outcomes [6.7]. purpose of this study was to evaluate the functional outcome of patients with Lisfranc joint injuries treated with fluoroscopically guided closed reduction and internal fixation with Kirschner wires within 24 hours of injury.

\section{Materials and Methods}

We performed a consecutive study of 20 patients with tarsometatarsal joint injuries at our hospital commencing in 2012 after approval of C-Arm for our emergency operation theatre. An informed consent was taken from each patient. Only those patients were included in this study that presented less than 24 hours of injury and were aged between 18 years and 50 years. The patients who were excluded from study included patients with open injuries, patients presenting more than 24 hours of injury, severe swollen feet requiring fasciotomies, difficult cases needing open reduction and polytrauma patients. All patients were evaluated by radiographs which included anteroposterior, oblique and lateral view of both feet and the injuries were classified as per Myerson's classification [8].

\section{Surgical Technique}

After appropriate anesthesia and draping closed reduction was achieved by holding fore foot and metatarsals and applying traction with assistant holding ankle/distal leg for contertraction. Valgus or varus manipulation was done for proper reduction of fracture along with dorsoplantar manipulation each guided by initial radiograph and subsequent fluoroscopic images. When satisfactory reduction in anteroposterior, lateral and oblique was confirmed kirschner wires were introduced percutaneouly to hold the reduction. Main focus was laid on second metatarsal cuneiform and first metatarsal, second cuneiform bones and the third metatarsocuneiform joint. The second metatarsal was reduced to the medial border of middle cuneiform; the joints were fixed with kirschner wires. The fourth and fifth metatarsals usually reduced once the above three reductions were achieved and were held with one or two transarticular K-wires from the base of 5th metatarsal to the cuboid. After satisfactory reduction and kirschner wire fixation pin site antiseptic dressing was done. A posterior plaster of Paris splint was applied in all caeses, which was converted to short leg cast after one week to avoid any cast complication that may arise due to postoperative swelling. K-wires were removed at 8 weeks. Full weight bearing was allowed at 10-12 weeks (Figures 3 and 4).

\section{Results}

Most patients $(70 \%, \mathrm{n}=14)$ were in age group of $18-35$ years with a mean 33.2 years. Males $(80 \%, n=16)$ outnumbered females $(20 \% n=4)$. Both right and left foot were almost equally involved. Cause of injury were road traffic accidents in $55 \%$ cases $(n=11)$, fall from height in $45 \%$ $(n=9)$. Metatarsal fracture was the most common associated injury $(30 \%$,

*Corresponding author: : Bhat SA, Government Medical College Srinagar, Srinagar, Jammu and Kashmir, India, Tel: 01346-244701; E-mail: shamim_asc@yahoo.com

Received January 24, 2015; Accepted February 28, 2014; Published March 10, 2014

Citation: Bhat SA, Kangoo KA, Zahoor A, Raja R, Jan S, (2015) Outcome after Fluoroscopic Guided Closed Reduction and Percutaneous Kirschner Wire Fixation of Lisfranc Joint Injuries: A Nationwide Randomized Controlled Trial. Surgery Curr Res 5: 220. doi:10.4172/2161-1076.1000220

Copyright: ( $) 2014$ Bhat SA, et al. This is an open-access article distributed under the terms of the Creative Commons Attribution License, which permits unrestricted use, distribution, and reproduction in any medium, provided the original author and source are credited. 


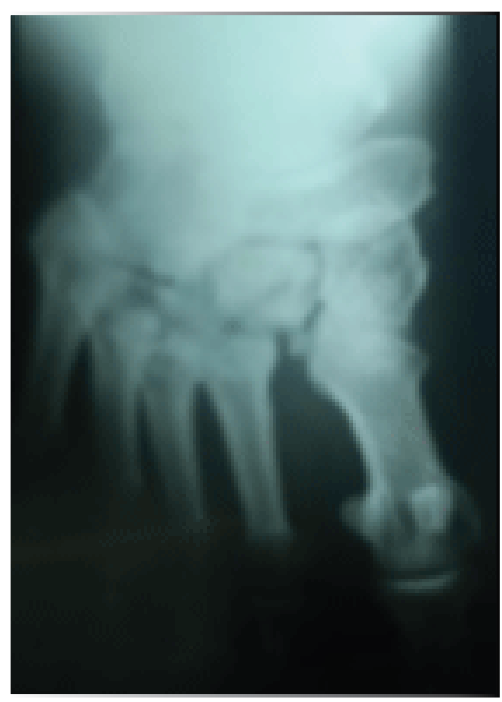

Figure 1: Preoperative radiograph.

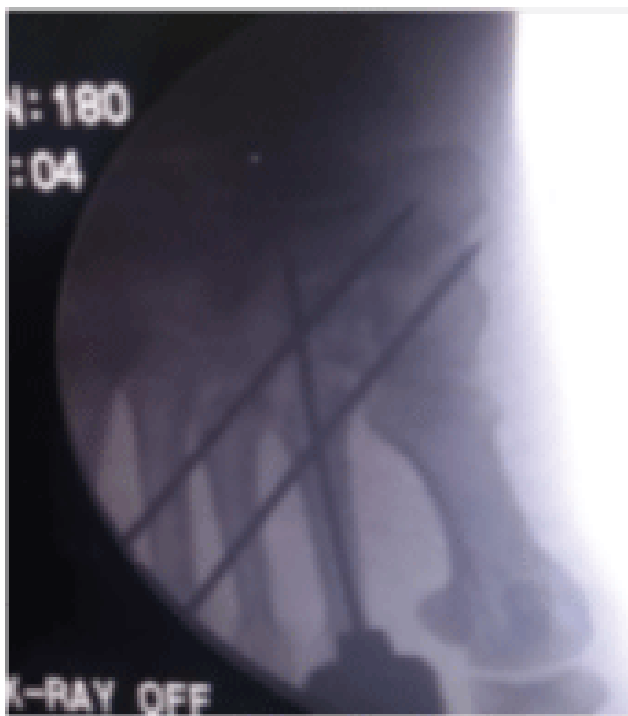

Figure 2: Flouroscopic assited reduction and fixation.

$\mathrm{n}=6$ ). The injuries were classified by Myerson's classification [8]. The majority of injuries $(60 \%, n=12)$ were type B followed by type A $(30 \%$, $\mathrm{n}=6)$. All the operations were performed within 24 hours of injury; with follow up from 9 months to 34 months with 12 (60\%) having final follow up of more than 22 months. Anatomical reduction was obtained in 19 patients (95\%). There was one case of loss of reduction and two cases of superficial pin site infection in our series both of them responded to oral antibiotics. There was no case of compartment syndrome of any foot in our series. Good to fair results were seen in $90 \%$ cases $(n=18)$. The mean AOFAS-M (American Orthopaedic Foot and Ankle Society Midfoot) score in our study was 78 with most patients perceiving mild chronic pain and decreased recreational activities. Eighty percent of patients were able to return to their original occupation, including 10 household or office workers and six laborers.

\section{Discussion}

Lisfranc injuries usually result from high-energy injuries, in our study motor vehicular accidents were the most common cause of injury, a finding consistent with the already available literature. Anatomic reduction and internal fixation has become standard principle governing treatment of tarsometatarsal fracture dislocations. Most authors agree stable anatomic reduction leads to optimal results [9] Controversy continues for method of fixation in Lanfranc joint injuries with proponents of both $\mathrm{k}$-wire fixation $[10,11]$, and screw fixation $[9,12]$. In our study the age ranged from $18-50$ years with average age of 33 years. In Goossens et al study [13], age groups ranged from 1052 years with mean of 34 while as reported in Pereira et al [14], age group ranged from 17-50 years with mean of 31.53. The mean age group in our study was close to the study of Goossens et al. [13]. Males outnumbered females in our study with ratio of 4:1 while as in Hesp et al. [15], the male to female ratio was 2.3:1. The reason for higher male to female ratio in our study may be due to the fact that most of females in our part of world are household workers. Both right and left feet were equally involved in our study (Figures 5 and 6).

The mode of injury was road traffic accidents (RTA) in majority of patients (55\%) followed by fall from height which was consistent with

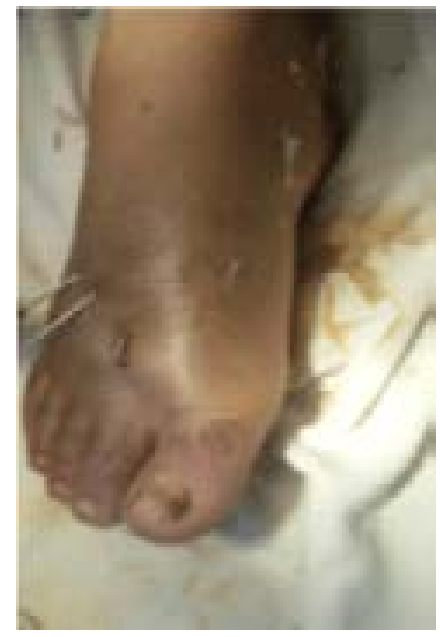

Figure 3: Percutaneous K-wire fixation.

Hardcastle et al [8], 40.3\% RTA and Kuo et al. [16], with RTA $42 \%$. In our study most of the Lisfranc injuries (60\%) were type Myerson type B followed by type A (30\%). In Enríquez et al. [17] series type B injuries were most common Lisfranc injuries (50\%), while as in Pereira et al series [14], type B Lisfranc joint injuries constituted 80.94 percent of Lisfranc fracture dislocations. Metatarsal fractures were the most common associated injury in our study in $30 \%$ cases. In Goossens et al., series [13] metatarsal injuries were also the most common associated injuries (40\%).

The mean duration of hospital stay in our series was three days. $\mathrm{K}$-wire were removed at mean of 8 weeks in our study while as in Kuo et al. [16] K-wires were removed at 6-8 weeks. Complication in our study included loss of reduction in one case and two cases of superficial pin site infection. Both cases occurred within one week of surgery and responded well to antibiotics and daily dressings. The percentage of loss of reduction with $\mathrm{K}$-wires was less in our series as we immobilized the foot for longer duration in short leg cast (average 8 weeks). Molded arch support was given to patients after three months, which was discarded 


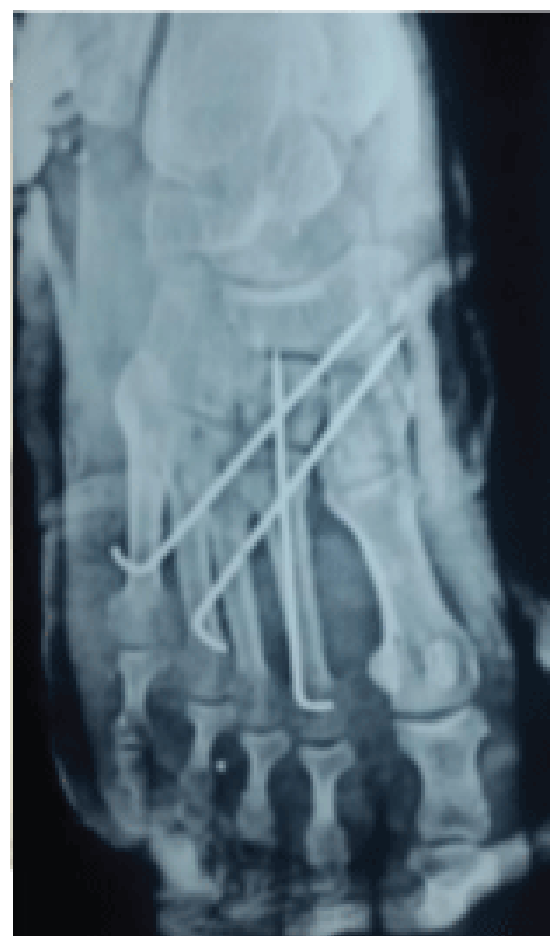

Figure 4: Postoperative antero-posterior radiograph.

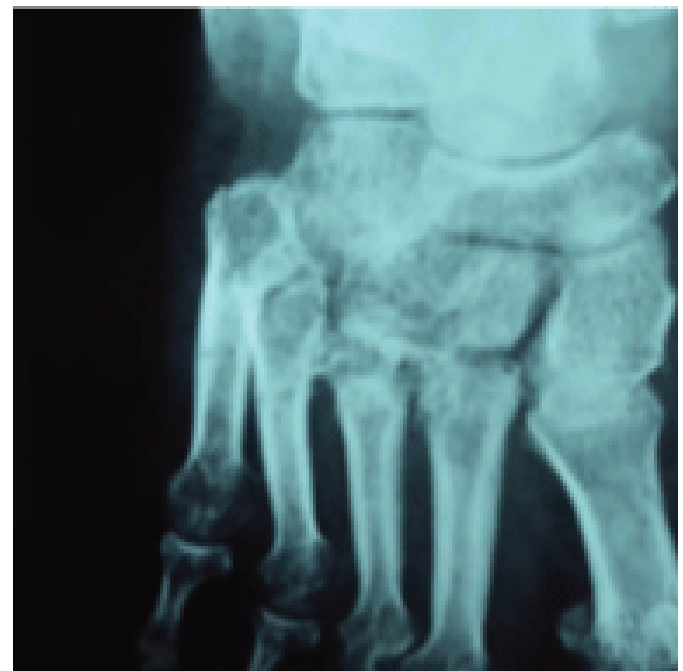

Figure 5: Final antero-posterior radiograph

at 6 months in $70 \%$ cases while as $30 \%$ cases felt its need up to one year. Good to fair results were seen in $90 \%$ cases as per scale used by Pereira et al. [14], with average AOFAS score 78, most of our patients

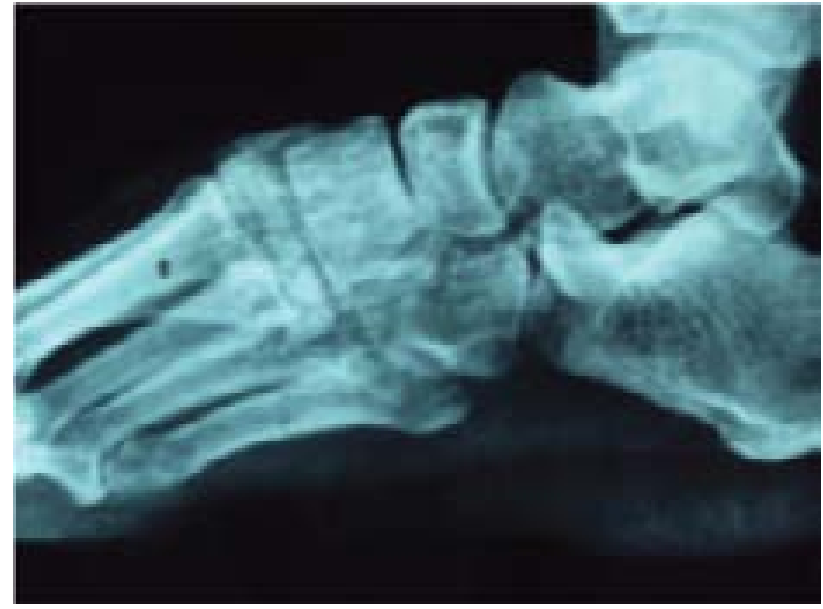

Figure 6: Final lateral foot radiographs

lost points to pain and decreased recreational activities.

We believe that early closed reduction and K-wire fixation considerably improves the functional outcome in these injuries. There is an added advantage that there is no scar related discomfort and cosmetic concern and no second surgery for removal of hardware is required. The disadvantage is that this method needs longer period of immobilization in a cast and chances of less precise anatomical reduction of Lisfranc joint. The limitation of our study is that there was no control group so that we could compare our results.

Further studies with large sample size and comparative studies are needed for definitive conclusions.

\section{References}

1. Cain PR, Seligson D (1981) Lisfranc's fracture-dislocation with intercuneiform dislocation: presentation of two cases an a plan for treatment. Foot Ankle 2: 156-160.

2. Norfray JF, Geline RA, Steinberg RI, Galinski AW, Gilula LA (1981) Subtleties of Lisfranc fracture-dislocations. AJR Am J Roentgenol 137: 1151-1156.

3. Kuo RS, Tejwani NC, Digiovanni CW, Holt SK, Benirschke SK, et al. (2000) Outcome after open reduction and internal fixation of Lisfranc joint injuries. J Bone Joint Surg Am 82: 1609-1618.

4. Arntz CT, Veith RG, Hansen ST (1988) Fractures and fracture-dislocations of the tarsometatarsal joint. J Bone Joint Surg Am 70: 173-181.

5. Buzzard BM, Briggs PJ (1998) Surgical management of acute tarsometatarsal fracture dislocation in the adult. Clin Orthop Relat Res 353: 125-33.

6. Curtis MJ, Myerson M, Szura B (1993) Tarsometatarsal joint injuries in the athlete. Am J Sports Med 21: 497-502.

7. Myerson M (1989) The diagnosis and treatment of injuries to the Lisfranc joint complex. Orthop Clin North Am 20: 655-664.

8. Myerson MS, Fisher RT, Burgess AR, Kenzora JE (1986) Fracture dislocations of the tarsometatarsal joints: end results correlated with pathology and treatment. Foot Ankle 6: 225-242.

9. Rosenberg GA, Patterson BM (1995) Tarsometatarsal (Lisfranc's) fracturedislocation. Am J Orthop

10. Suppl : 7-16.

11. Pérez blanco R, Rodríguez Merchán $C$, Canosa Sevillano R, Munuera Martínez $L$ (1988) Tarsometatarsal fractures and dislocations. J Orthop Trauma 2: 188194. 
Citation:Bhat SA, Kangoo KA, Zahoor A, Raja R, Jan S, (2015) Outcome after Fluoroscopic Guided Closed Reduction and Percutaneous Kirschner Wire Fixation of Lisfranc Joint Injuries: A Nationwide Randomized Controlled Trial. Surgery Curr Res 5: 220. doi:10.4172/2161-1076.1000220

12. Tan YH, Chin TW, Mitra AK, Tan SK (1995) Tarsometatarsal (Lisfranc's) injuries-results of open reduction and internal fixation. Ann Acad Med Singap 24: 816819

13. Jeffreys TE (1963) Lisfranc's fracture-dislocation: a clinical and experimental study of tarso-metatarsal dislocations and fracture-dislocations. J Bone Joint Surg $\mathrm{Br}$ 45: 546-551.

14. Goossens M, De stoop N (1983) Lisfranc's fracture-dislocations: etiology, radiology, and results of

15. treatment. A review of 20 cases. Clin Orthop Relat Res. 176: 154-162

16. Pereira CdJ, Espinosa EG, Miranda I, Pereira MB, Canto RSdT (2008)
Evaluation of the surgical treatment of Lisfranc joint fracture-dislocation. Acta ortop bras 16: 93-97.

17. Hesp WL, Van der werken C, Goris RJ (1984) Lisfranc dislocations: fracture and/or dislocations through the tarso-metatarsal joints. Injury 15: 261-266.

18. Kuo RS, Tejwani NC, Digiovanni CW, Holt SK, Benirschke SK, et-al. (2000) Outcome after open reduction and internal fixation of Lisfranc joint injuries. Bone Joint Surg Am 82: 1609-1618.

19. Enríquez CJA, López VA, García HA, González TA, Ventura MA, et al. (2005) Lisfranc's fracture dislocation. Epidemiological study and results at the General Hospital in Mexico. Acta Ortop Mex 19: s1. 